\title{
Longitudinal Change Detection: Inference on the Diffusion Tensor Along White-Matter Pathways
}

\author{
Antoine Grigis ${ }^{1,2, \star}$, Vincent Noblet ${ }^{1}$, Fréderic Blanc ${ }^{2}$, Fabrice Heitz ${ }^{1}$, \\ Jérome de $\mathrm{Seze}^{2}$, and Jean-Paul Armspach ${ }^{2}$ \\ 1 University of Strasbourg, LSIIT, UMR 7005, CNRS, France \\ 2 University of Strasbourg, LINC-IPB, UMR 7237, CNRS, France
}

\begin{abstract}
Diffusion tensor magnetic resonance imaging (DT-MRI) tractography allows to probe brain connections in vivo. This paper presents a change detection framework that relies on white-matter pathways with application to neuromyelitis optica (NMO). The objective is to detect global or local fiber diffusion property modifications between two longitudinal DT-MRI acquisitions of a patient. To this end, estimation and testing tools on tensors along the white-matter pathways are considered. Two tests are implemented: a pointwise test that compares at each sampling point of the fiber bundle the tensor populations of the two exams in the cross section of the bundle and a fiberwise test that compares paired tensors along all the fiber bundle. Experiments on both synthetic and real data highlight the benefit of considering fiber based statistical tests compared to the standard voxelwise strategy.
\end{abstract}

\section{Introduction}

Diffusion weighted magnetic resonance imaging (DW-MRI) is a non-invasive method for characterizing the diffusion of water molecules in tissues. The automated detection of relevant changes in longitudinal DW-MRI sequences may open promising perspectives for medical diagnosis, follow-up and prognosis. Diffusivity profiles obtained from DW-MRI acquisitions are usually modeled by diffusion tensors of rank 2 (DTs). Some previous work has addressed change detection between DT-derived scalar images, [2], [4], or between DT fields, [3], 6], [5]. In [6], statistical tests have been developed to compare two sets of tensors with application to the comparison of two groups of subjects. Applying these tests in the context of the longitudinal analysis of a given subject requires to extract at each voxel two populations of tensors to be compared. This step is not straightforward since we need to ensure that all the tensors are drawn from the same distribution. A natural idea is to learn tensor distribution at each voxel by considering all the tensors in a surrounding user-defined spatial neighbourhood. This is based on the commonly made assumption of a constant piecewise model, with known limitations, in particular, at the interface between tissues. To circumvent this limitation, a local bootstrap strategy can be used to generate a set

* grigis@unistra.fr

G. Fichtinger, A. Martel, and T. Peters (Eds.): MICCAI 2011, Part II, LNCS 6892, pp. 1-8, 2011.

(C) Springer-Verlag Berlin Heidelberg 2011 


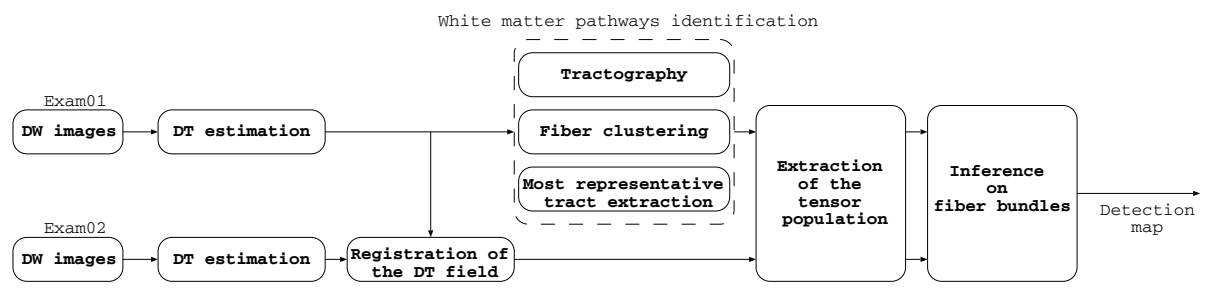

Fig. 1. Flowchart of the proposed framework

of tensors characterizing the variability of each voxel, based on the variability existing in the DW-MRI images [5].

In this paper we suggest a longitudinal DT change detection framework based on tractography features (Fig. 1). The construction of a neighbourhood based on the fiber structures, and the application of statistical tests along the fiber, enable the detection of subtle changes along white-matter pathways. The proposed processing pipeline is as follows. First, eddy current distortions are corrected using FSL 1 . DTs are then estimated from the DW images using a least square algorithm. Then, a fiber tract extraction is performed with Slicen 2 (Label Seeding module) exploiting the first exam information. Fibers of the first exam are clustered to generate homogeneous fiber bundles (section 2.1). The most representative tract of each bundle is extracted and used as a reference tract for the bundle (section 2.2). The DTs of the second exam are warped using a linear interpolation in the Log-eulidean space and the preservation of principal direction (PPD) reorientation strategy to be aligned with the first exam [5]. To this end, an affine and a Bspline based transformation are estimed from the FA images of the two acquisitions [5]. Tensor sets reflecting the local variability are extracted at each sampling point of the reference tract considering the DTs of the two registered exams that belong to the cross section of the bundle (section 3.1). Finally local and global multivariate statistical tests along white-matter principal pathways are applied (section 3.2 ). Experiments on synthetic data highlight the benefit of considering fiber based statistical tests compared to a standard voxelwise strategy. Application to neuromyelitis optica (NMO) also demonstrates the clinical relevance of the fiber-based strategy.

\section{Identifying White-Matter Fiber Bundles in DT Data}

\subsection{Fiber Clustering}

Tractography methods produce a dense set of curves that bear a close resemblance to known white-matter pathways [7. Considering a method that automatically clusters and labels these curves into anatomically plausible pathways

\footnotetext{
${ }^{1}$ http://www.fmrib.ox.ac.uk/fsl/

2 http://www.slicer.org/
} 
is of great interest for a relevant analysis of white matter properties. To this end, we have implemented a fiber bundle clustering algorithm based on the method described in [7. Neighboring fiber tracts are grouped using the mean of thresholded closest distance. With this metric the distance between curves $Q$ and $R$ is expressed as follows:

$$
d_{t}(Q, R, t)=\text { mean }_{a \in Q,\|a-b\|>t} \min _{b \in R}\|a-b\|
$$

where $\mathrm{t}$ is the threshold below which the distances are discarded. This threshold enables the separation of two fibers that might run very closely together for a long course and then diverge abruptly for a relatively short course into two different clusters [7. Since this metric is not symmetric, we consider the longer mean of thresholded closest distances in the clustering algorithm (see [7] for a discussion on the influence of the chosen symmetrized form):

$$
d_{L t}(Q, R, t)=\max \left(d_{t}(Q, R, t), d_{t}(R, Q, t)\right)
$$

With this metric it is possible to identify corresponding tracts with high consistency even when the global shapes of the tracts differ markedly in length or curvature [7]. A single-linkage agglomerative clustering is then performed [7]. The number of clusters is inversely related to a user-defined fusion proximity threshold $d_{f}$. The main problem of the tract-clustering algorithm is the computational burden related to the calculation of the pairwise distances between the current fiber and all the previously clustered fibers. To circumvent this issue, pairwise distances are only computed for fibers belonging to clusters whose center of mass are close enough to the center of mass of the current fiber (i.e., specification of a user-defined distance threshold $\left.d_{c m}\right)$. After the clustering step, bundles with less than $N_{c}$ fibers are rejected in order to have a minimum number of samples in the statistical population when a pointwise test is performed.

\subsection{Bundle Most Representative Tract}

We denote each tract belonging to the $i^{\text {th }}$ bundle $T_{i, j}$, where $n_{i}$ is the number of tracts in the bundle $i$ and $j \in\left\{1, \ldots, n_{i}\right\}$. Then the most representative tract $T_{\text {ref,i } i}$ of the $i^{\text {th }}$ bundle is obtained by selecting the fiber tract that minimizes the sum of distances with other tracts. The metric used here is the average mean of closest distances:

$$
d_{M C}(Q, R)=\frac{d_{t}(Q, R, 0)+d_{t}(R, Q, 0)}{2}
$$

Note that we set $t=0$ in the thresholded distance $d_{t}$ since we are dealing with a coherent bundle. Finally, the criterion used to select the most representative tract of a bundle $i$ can be summarized as follows:

$$
T_{r e f, i}=\arg \min _{T_{i, k}, k \in 1, \ldots, n_{i}}\left[\sum_{l \in 1, . ., n_{i}} d_{M C}\left(T_{i, k}, T_{i, l}\right)\right]
$$

Each most representative tract $T_{r e f, i}$ is then resampled with $\mathrm{N}$ points ( $\mathrm{N}$ being user-defined) using a cardinal basis spline interpolation. 

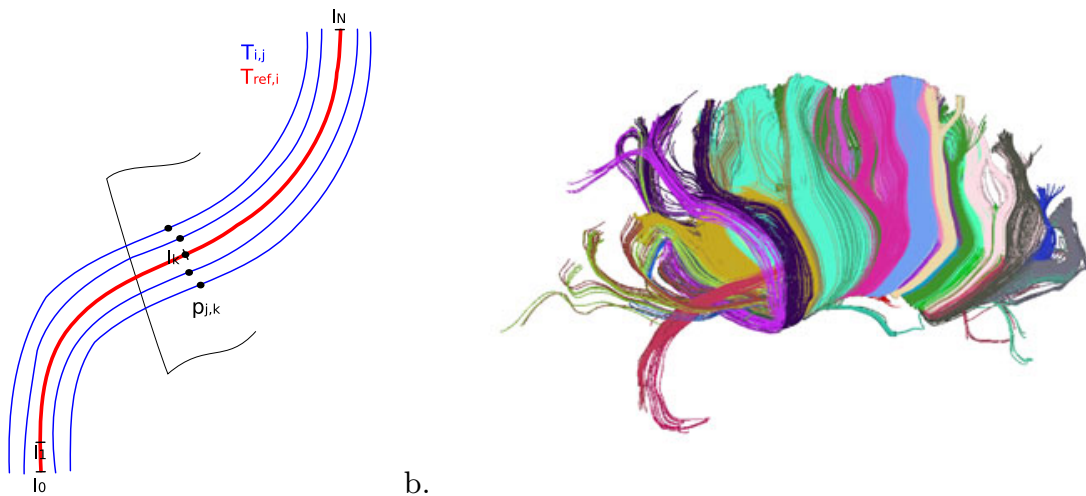

b.

Fig. 2. a. Schematic representation of the extraction of the tensor sets reflecting the local variability: see text, and b. the result of the clustering step on a tractography obtained with Slicer from a ROI in the corpus callosum (parameters: see text).

\section{Inference on Fiber Bundles}

\subsection{Extraction of Tensor Populations Reflecting the Local Variability}

Inference on DT is composed of an estimation and a testing step. The estimation of model parameters requires to consider a population of tensors that are all assumed to be drawn from the same distribution. In many situations, considering all the tensors in a given spatial neighbourhood may not satisfy this assumption, especially at the interface between different anatomical structures. To overcome this problem we propose to select a tensor population by taking advantage of the local geometry of the bundles (see Fig. 2 a). For each sampling point $l_{k}$ of the reference tract $T_{r e f, i}$, a population of tensors is extracted by considering the tensors located at the intersections $p_{j, k}$ between the plane perpendicular to $T_{r e f, i}$ and each fiber $T_{i, j}$ of the bundle. Since points $p_{j, k}$ may not lie on the grid, corresponding tensors are obtained using linear interpolation in the Logeuclidean space. In some cases, the plane might intersect the bundle in several points (e.g. for U-shaped bundles). Consequently, only the closest intersection is considered. Besides to remove outliers, intersections $p_{j, k}$ whose distance to $l_{k}$ is larger than $3 \sigma_{i}$ are discarded, $\sigma_{i}$ being the median absolute distance of the $p_{j, k}$ 's to their corresponding sampling point $l_{k}$ over the $i^{\text {th }}$ bundle.

\subsection{Multivariate Statistical Estimation and Testing on Tensors}

Many statistical tests rely on the normal distribution. Considering the multivariate normal distribution for the symmetric positive definite (SPD) matrices has the drawback to associate matrices with negative or null eigenvalues with a non null probability. To circumvent this limitation, Schwartzman suggests to model the matrix logarithms with the multivariate normal distribution, which 
comes to model the SPD matrices with a Log-normal distribution 6]. To this end the Log-euclidean metric is used. Note that the logarithm of an SPD matrix $\mathrm{D}$ is obtained as $L=\log (D)=U \log (\Lambda) U^{T}$, where $\Lambda$ and $U$ are the matrices derived from the standard spectral decomposition, containing respectively the eigenvalues and the eigenvectors of D. Then, the Log-euclidean distance between two SPD matrices $D_{1}$ and $D_{2}$ can be defined as the Euclidean distance between their logarithms $d^{2}\left(D_{1}, D_{2}\right)=\left\|\log \left(D_{1}\right)-\log \left(D_{2}\right)\right\|^{2}$. According to this metric, an estimator of the mean $\bar{D}$ of a set of $\mathrm{n}$ tensors $D_{i}$ is given by the exponential of the arithmetic Log-tensors mean, i.e. $\bar{D}=\exp (\bar{L})$, with:

$$
\bar{L}=\operatorname{argmin}_{\Sigma} \sum_{i=1}^{n}\left\|\log \left(D_{i}\right)-\Sigma\right\|^{2}=\frac{1}{n} \sum_{i=1}^{n} \log \left(D_{i}\right)
$$

An estimator of the variance is then:

$$
s^{2}=\frac{1}{6(n-1)}\left[\sum_{i=1}^{n} \operatorname{tr}\left(\log \left(D_{i}\right)-\bar{L}\right)^{2}\right]
$$

Pointwise testing. Based on this model, it is possible to derive a statistical test on tensor's eigenvalues. We consider two populations of $n_{1}$ and $n_{2}$ tensors respectively $\left(n=n_{1}+n_{2}\right)$. Under the assumption that the tensor logarithms of the two populations follow the normal distributions $L_{1} \sim \mathcal{N}\left(M_{1}, \sigma^{2} I d\right)$ and $L_{2} \sim \mathcal{N}\left(M_{2}, \sigma^{2} I d\right)$, the maximum likelihood estimates of $M_{1}, M_{2}$, and $\sigma^{2}$ are respectively $\overline{L_{1}}, \overline{L_{2}}(\mathrm{Eq} 5)$, and $\hat{\sigma}^{2}=\left[\left(n_{1}-1\right) s_{1}+\left(n_{2}-1\right) s_{2}\right] /(n-2)(\mathrm{Eq}[6)$. We consider a test [6] that evaluates whether the two populations of diffusion tensors have similar eigenvalues, but possibly different eigenvectors. Let $\Lambda_{1}, U_{1}$ and $\Lambda_{2}, U_{2}$ be the matrices derived from standard spectral decomposition, and containing respectively the eigenvalues and eigenvectors of $M_{1}$ and $M_{2}$. The test, based on the log-likelihood ratio under hypotheses $H_{0}: \Lambda_{1}=\Lambda_{2}$ vs $H_{1}: \Lambda_{1} \neq \Lambda_{2}$ with $U_{1} \neq U_{2}$ unknown is:

$$
T_{\text {pointwise }, \sigma}=\frac{n_{1} n_{2}}{3 n^{2} \hat{\sigma}^{2}} \operatorname{tr}\left[\left(\bar{\Lambda}_{1}-\bar{\Lambda}_{2}\right)^{2}\right]
$$

where $\overline{\Lambda_{1}}$ and $\overline{\Lambda_{2}}$ are the eigenvalue matrices of $\overline{L_{1}}$ and $\overline{L_{2}}$, respectively. We investigate in the sequel the influence of estimating the variance $\sigma^{2}$ locally for each sampling point $l_{k}$ or of considering a constant variance $\sigma_{b_{i}}^{2}$ estimated over

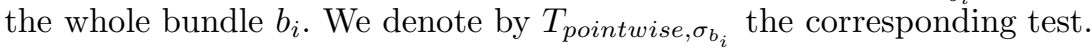

Fiberwise testing. It may be of great interest for physicians or neuroscientists to identify global modifications along fiber bundles. To this end, we propose to build a list of putative evolving fibers ordered by decreasing evolution probability. At each of the $\mathrm{N}$ sampling points of the most representative tracts $T_{\text {ref, } i}$, the difference between the fiber cross section mean Log-tensors are computed. A population of $N$ Log-tensors is thus obtained for each bundle. Under the assumption that the Log-tensor population follows the normal distribution $L \sim \mathcal{N}\left(M, \sigma^{2} I d\right)$, the maximum likelihood estimates of $M$, and $\sigma^{2}$ are respectively $\bar{L}$ (Eq $\sqrt[5]{5}$ ), and 
$\hat{\sigma}^{2}=s^{2}$ (Eq 6 ). We consider a test that evaluates whether the population of Log-tensors has eigenvalues equal to $\Lambda_{0}=[0,0,0][$ [ . Let $\Lambda, U$ be the matrices derived from standard spectral decomposition, and containing respectively the eigenvalues and eigenvectors of $M$. The test, based on the log-likelihood ratio under hypotheses $H_{0}: \Lambda=\Lambda_{0}$ vs $H_{1}: \Lambda \neq \Lambda_{0}$ is:

$$
T_{\text {fiberwise }}=\frac{N}{\hat{\sigma}^{2}} \operatorname{tr}\left[\left(\bar{\Lambda}-\Lambda_{0}\right)^{2}\right]
$$

where $\bar{\Lambda}$ is the eigenvalue matrice of $\bar{L}$.

\section{Results}

Experiments on simulated changes. We consider two repeated DW-MRI acquisitions of the same healthy subject acquired on a 3T SIEMENS MRI scanner with 30 encoding gradients (b-value of $1000 \mathrm{~s} / \mathrm{mm}^{2}$ ). In this way, the differences between the two scans are only due to the acquisition noise and distortion. The image dimensions are $96 \times 96 \times 55$ and the spatial resolution is $2 \times 2 \times 2 \mathrm{~mm}^{3}$. The tractography was computed for the first image using the Label Seeding module in Slicer (integration step length of $0.5 \mathrm{~mm}$, minimum tract length of $50 \mathrm{~mm}$, and stopping values on the fractional anisotropy and curvature of 0.1 and 0.8 respectively). For the clustering step, a fusion threshold $d_{f}$ of $3.5 \mathrm{~mm}$, a cut-off threshold $t$ in the metric of $0.5 \mathrm{~mm}$, and a selection threshold $d_{m c}$ of $4 d_{f}$ were used. Results are presented in Fig. 2 $\mathrm{tb}$ where each bundle contains at least $N_{c}=9$ fibers. A synthetic fiber alteration is simulated in one of the two scans as follows. After the fiber clustering step, a bundle is selected to generate a modification mask. Inside the modification mask, the diffusivity in the principal direction, i.e. the principal eigenvalue, is uniformly modified by applying a multiplicative factor $k \in] 1,1.5]$. The results of the pointwise test are compared with the $3 \times 3 \times 3$ spatial neighbourhood approach (SN) described in [5]. To this end, we use a projection $P_{1}$ from the ijk coordinate system to the fiber coordinate system using the nearest neighbour interpolation. The criterion used to compare the different methods is the area under Receiver Operating Characteristic (ROC) curves. A test that allows a perfect discrimination is characterized by ROC plot with an area of one. A resampling of the most representative fiber in $N=200$ points is done before the bundle cross section point extraction. The Table 1 summarizes the results. The pointwise methods outperform the SN method, thus pointing out the importance of satisfying the constant piecewise assumption. Estimating a constant variance for each bundle slightly increases the performance of the statistical test. The sensitivity of the fiberwise test $T_{\text {fiberwise }}$ is also explored (Table 11). Notice that in this particular case the fiberwise test is more appropriate and gives better results since a global modification has been simulated.

Experiments on NMO patients. Neuromyelitis Optica (NMO) is an inflammatory disease of the central nervous system that predominantly affects optic 
Table 1. Areas under the ROC curves (diffusion modification: see text)

\begin{tabular}{|c|c|c|c|c|c|c|c|c|c|c|c|c|c|}
\hline $\mathrm{k}$ & 1.01 & 1.02 & 1.03 & 1.04 & 1.05 & 1.06 & 1.07 & 1.08 & 1.09 & 1.1 & 1.2 & 1.3 & 1.4 \\
\hline $\mathrm{SN}$ & 0.581 & 0.587 & 0.622 & 0.663 & 0.701 & 0.736 & 0.767 & 0.795 & 0.822 & 0.843 & 0.937 & 0.957 & 0.965 \\
$T_{\text {pointwise }, \sigma}$ & 0.698 & 0.707 & 0.724 & 0.746 & 0.705 & 0.793 & 0.815 & 0.834 & 0.852 & 0.868 & 0.953 & 0.979 & 0.989 \\
$T_{\text {pointwise } \sigma_{b_{i}}}$ & 0.721 & 0.727 & 0.745 & 0.771 & 0.801 & 0.832 & 0.859 & 0.884 & 0.905 & 0.921 & 0.986 & 0.996 & 0.998 \\
$T_{\text {fiberwise }}$ & 0.605 & 0.837 & 0.977 & 1.0 & 1.0 & 1.0 & 1.0 & 1.0 & 1.0 & 1.0 & 1.0 & 1.0 & 1.0 \\
\hline
\end{tabular}

a.

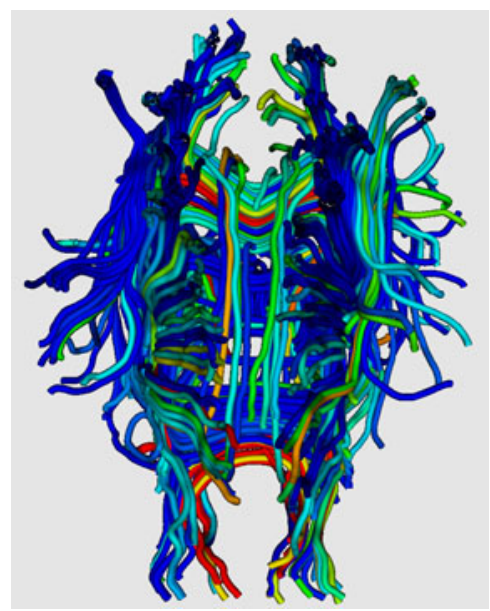

b.

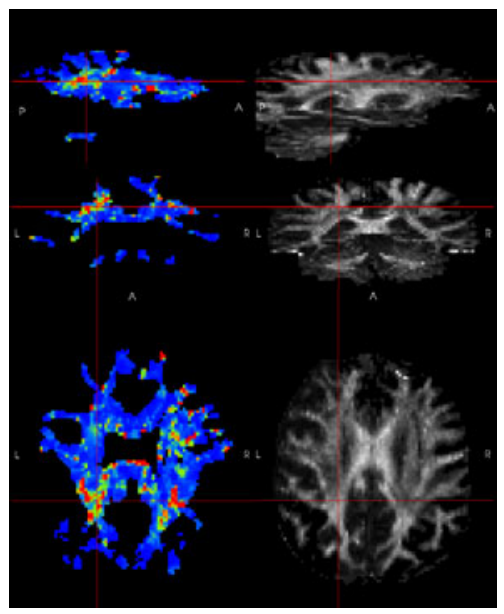

Fig. 3. a. fiberwise test (first patient) and b. projection of the pointwise test from the fiber coordinate system to the ijk coordinate (second patient).

nerves and spinal cord. The frequency of brain lesions is small using standard sequences of MRI (T1, T2 and T2-FLAIR). However, a recent work has demonstrated that the majority of NMO patients have cognitive impairment, which is a subcortical impairment [1. In the first part of this preliminary study, we compared the cognitive functions outcome of patient 1 (using the French translation of the Brief Repeatable Battery (BRB-N), a cognitive battery with 14 subtests) and brain diffusion MRI outcome (using the fiberwise test) done at the same time at M0 and M18. In the second part, we compared the neurological physical status outcome of patient 2 (using the expanded disability status scale (EDSS)) to brain diffusion MRI outcome (using the pointwise test) done at the same time at M0 and M18. In this latter in order to simplify the interpretation of the result, the fiber detections were transformed back to the voxel space. Images were acquired on a $1.5 \mathrm{~T}$ SIEMENS MRI scanner with 30 encoding gradients (b-value of $1000 \mathrm{~s} / \mathrm{mm}^{2}$ ) at 18 months apart. The images dimensions are $128 \times 128 \times 41$ and the spatial resolution is $1.8 \times 1.8 \times 3.5 \mathrm{~mm}^{3}$. For the first patient the fiberwise test was applied (Fig. 3-a). Two regions stand out: the anterior and posterior parts of the corpus callosum. These modifications are in accordance with cognitive and behavioral status that worsened. Patient 1 had 6 subtests out of 14 
inferior to the 5 th percentile at M0, and 7 subtests at M18. The second patient had a huge decrease of capacity to walk between M0 and M18. Thus, the EDSS increased from 6.5 (capable to walk with a constant bilateral assistance, such as canes) to 8.5 (essentially restricted to bed much of day, not capable to walk). This aggravation was in accordance with the results of the pointwise test showing modifications in the corticospinal tracts (Fig. 3-b).

\section{Conclusions}

The proposed multivariate statistical tests along white-matter principal pathways provide complementary information. The global test enables the identification of the fibers involved in the longitudinal evolution of the pathology, while the local strategy enables the detection of more subtle changes. Moreover, we have demonstrated the superiority of such methods compared to the standard voxelwise strategy. Finally, the proposed approach might open promissing perspectives for the follow-up of the NMO pathology, and will give way to further explorations. In the future we want to investigate whether a joint fiber clustering of the two exams would lead to a better mapping of the whitte matter tracts. We also want to study how registration accuracy could affect the statistical tests and investigate the impact of using a symmetric registration.

\section{References}

1. Blanc, F., Zéphir, H., Lebrun, C., Labauge, P., Castelnovo, G., Fleury, M., Sellal, F., Tranchant, C., Dujardin, K., Vermersch, P., de Seze, J.: Cognitive functions in neuromyelitis optica. Arch. Neurol. 65(1), 84-88 (2008)

2. Boisgontier, H., Noblet, V., Heitz, F., Rumbach, L., Armspach, J.P.: An automatic method for change detection in serial DTI-derived scalar images. In: Workshop MIAMS - MICCAI (2008)

3. Boisgontier, H., Noblet, V., Heitz, F., Rumbach, L., Armspach, J.P.: Generalized likelihood ratio tests for change detection in diffusion tensor images. In: ISBI, pp. 811-814. IEEE Press, Piscataway (2009)

4. Chung, S., Pelletier, D., Sdika, M., Lu, Y., Berman, J.I., Henry, R.G.: Whole brain voxel-wise analysis of single-subject serial DTI by permutation testing. Neuroimage 39(4), 1693-1705 (2008)

5. Grigis, A., Noblet, V., Renard, F., Heitz, F., Armspach, J.-P., Rumbach, L.: Change detection in diffusion MRI using multivariate statistical testing on tensors. In: Jiang, T., Navab, N., Pluim, J.P.W., Viergever, M.A. (eds.) MICCAI 2010. LNCS, vol. 6362, pp. 117-124. Springer, Heidelberg (2010)

6. Schwartzman, A.: Random ellipsoids and false discovery rates: statistics for diffusion tensor imaging data. Ph.D. thesis, Stanford University (2006)

7. Zhang, S., Correia, S., Laidlaw, D.H.: Identifying white-matter fiber bundles in DTI data using an automated proximity-based fiber-clustering method. IEEE Transactions on Visualization and Computer Graphics 14, 1044-1053 (2008) 